\title{
Single event latch-up detection for nano-satellite external solar radiation mitigation system
}

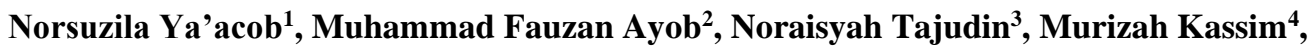 \\ Azita Laily Yusof ${ }^{5}$ \\ 1,2,3,4,5Faculty of Electrical Engineering, Universiti Teknologi MARA, Malaysia \\ ${ }^{1,5}$ Wireless Communication Technology, Faculty of Electrical Engineering, \\ Universiti Teknologi MARA, Malaysia
}

\begin{tabular}{l}
\hline \hline Article Info \\
\hline Article history: \\
Received Jun 9, 2016 \\
Revised Nov 20, 2016 \\
Accepted Dec 11, 2016 \\
\hline
\end{tabular}

\section{Keywords:}

Cubesat

Electrical power subsystem Single event effect (SEE)

Single event latch-up (SEL)

Single event upset (SEU)

\begin{abstract}
This paper presents the single event latch-up (SEL) detection for nano-satellite external solar radiation mitigation system. In this study, the SEL detection analysis was conducted using circuit test and simulation. An electrical power subsystem (EPS) is a part of all CubeSat bus subsystems and it comprises solar arrays, rechargeable batteries, and a power control and distribution unit (PCDU). In order to extract the maximum power generated by the solar arrays, a peak power tracking topology is required. This may lead to the SEL with the presence of high voltage produced by solar. To overcome the SEL problems, the circuit test and simulation must be done so that the flow of SEL will be easily detected and mitigate. The method that been used are by using microcontroller, the SEL will be created in the certain time. The programable integrated circuit (PIC) are used to mitigate SEL effect. It indicates that, the SEL occur very fast in certain time. When the simulation is conducted by using SPENVIS, the result shows, only single event upset (SEU) was affected on UiTMSAT-1.
\end{abstract}

This is an open access article under the CC BY-SA license.

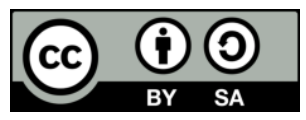

\section{Corresponding Author:}

Norsuzila Ya'acob,

Department of Faculty of Electrical Engineering,

UniversitiTeknologi MARA,

40450 Shah Alam, Selangor, Malaysia.

Email: norsuzila@uitm.edu.my

\section{INTRODUCTION}

On August $10^{\text {th }}$ 2018, UiTMSAT-1 the first UiTM's nanosatellite has successfully made their launch to the space from the international space station (ISS). This BIRDS-2 Cubesat nanosatellite along with the other 2 Cubesats, MAYA-1 from the Philippines and BHUTAN-1 from Bhutan is orbiting in low earth orbit (LEO) at the altitude of approximately $400 \mathrm{~km}$. The nanosatellite is currently orbiting around the Earth with the velocity of $7.66 \mathrm{~km} / \mathrm{s}$ and execute the transmission of its beacon to the UiTM ground station. The UiTM ground station is situated at the Faculty of Electrical Engineering, Tower 2, Engineering Complex, UiTM Shah Alam [1-3].

When it comes to LEO path, there are several radiations that is present. This radiation may lead to some single event effect (SEE) which consists of single event upset (SEU), single event-latchup (SEL), single event burnout (SEB), and single event gate rupture (SEGR) [4-9]. This paper is focusing on SEL effects and mitigating it. In complementary metal-oxide-semiconductor (CMOS) technologies or in some sillicon on insulator (SOI) when n- and p-type devices are built into common silicon islands, SEL presents in bulk. It has been found in space cosmic ray environments and terrestrial neutron accelerated tests. In addition, SEL may generate non-catastrophic interconnect damage from melting [10-14]. 
The SEL results in the maneuvering satellite platform (MSP) and programable integrated circuit (PIC) devices in radiation test. Furthermore, it commonly did not result in failed devices although drawing high current. In consequence, there is always a danger of latent failures. Tested MSP devices were found to not operate properly. after $1 \times 106 / \mathrm{cm}^{2}$ at $86 \mathrm{MeV}-\mathrm{cm}^{2} / \mathrm{mg}$ linear energy transfer (LET). On the contrary, devices such as PIC, Atmel, Intel and Qualcomm devices were observed to operate properly (after powder cycle) after exposure to more than $1 \times 107 / \mathrm{cm}^{2}$ at LETs of at least $75 \mathrm{MeV}-\mathrm{cm}^{2} / \mathrm{mg}$. It is crucial to have a stable and reliable power system for a Cubesat to be functioning. The Cubesat will not be able to do anything else without electrical power other than drifting around in orbit. Hence, the EPS subsystem contributes a primary role in this case. The current must be limited to protect the subsystem if the EPS draws a current that is immense enough to suggest a malfunction [15]. The SEL might occur to the spacecraft which is in this case, Cubesat, caused by heavy ion or protons from cosmic rays or solar flares during the operation. Even small increment of current over normal supplied current, the SEL will occur and may damage the systems overall. [16-23].

This paper presents on simulating SEL event by using radiation in substitute of using microcontroller. Also using PIC to detect and mitigate the SEL that occur as replicate to nano-satellite. The objective for this research puts its emphasis on SEL that takes place to the system through the solar panel and collecting the data during normal current and during the SEL occurred. Latch-up is destructive and a power cycle is required to restore the operation. A significant vulnerability in CMOS space systems happens as a result of the latch-up that is stimulated by ionizing radiation such as high-energy proton or heavy-ions from deep space. The sensitivity of an integrated circuit (IC) to SEL relies on assorted process parameters as well as design geometry [24]. Nevertheless, it can be corrected if the SEL is detected and the device's power is quickly turned off, then turned back on. So, the detection of SEL must be detected faster before its occurrence to avoid the harm to the other components. The SEL can take place in split second at any time at certain temperature $[25,26]$ especially in space. The mitigation of the SEL must be there as well so that the device can operate normally and continue with the main missions without any trouble.

\section{RESEARCH METHOD}

\subsection{Block diagram}

Figure. 1 shows that the SEL is occurring in the gray box. This is where the radiation from space with several amount of energy that can cause the SEL effect. The mitigation must be done before the radiation starts to damage the OBC. In this case, the system must be turn off immediately and turn it back on [27]. This is where the reset PIC take place in order to reset the system.

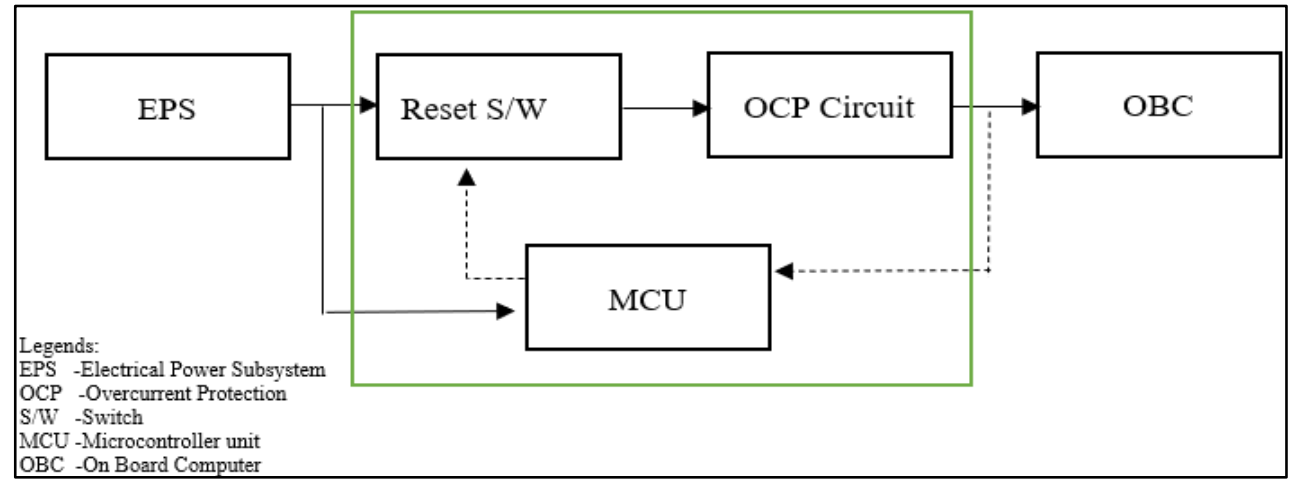

Figure1. Block diagram of reset PIC placed between EPS and OBC

By using PIC16F1787, it may protect the OBC from harm of SEL. It is functioned to reset the systems. The PIC was named as reset PIC. First, the process starts with the normal current supply to the PIC. In this state, there is nothing happened and the flow of current smooth. The current flow straight to the overvoltage protection which is used here is LTC4361CTS81. After a several time, the high current will be injected to the process which is in this case it is equal to SEL occurrence. The overcurrent protection will detect the high current and will reset the flow of the current so that the board is not receive any damage. After a few periods, the process will turn back on and flow the normal current back to the on-board computer. Figure 2 shows the flowchart of mitigating the SEL. While Figure 3 shows the EPS standard block diagram used in this system to analyze the SEL effect in Cubesats. 


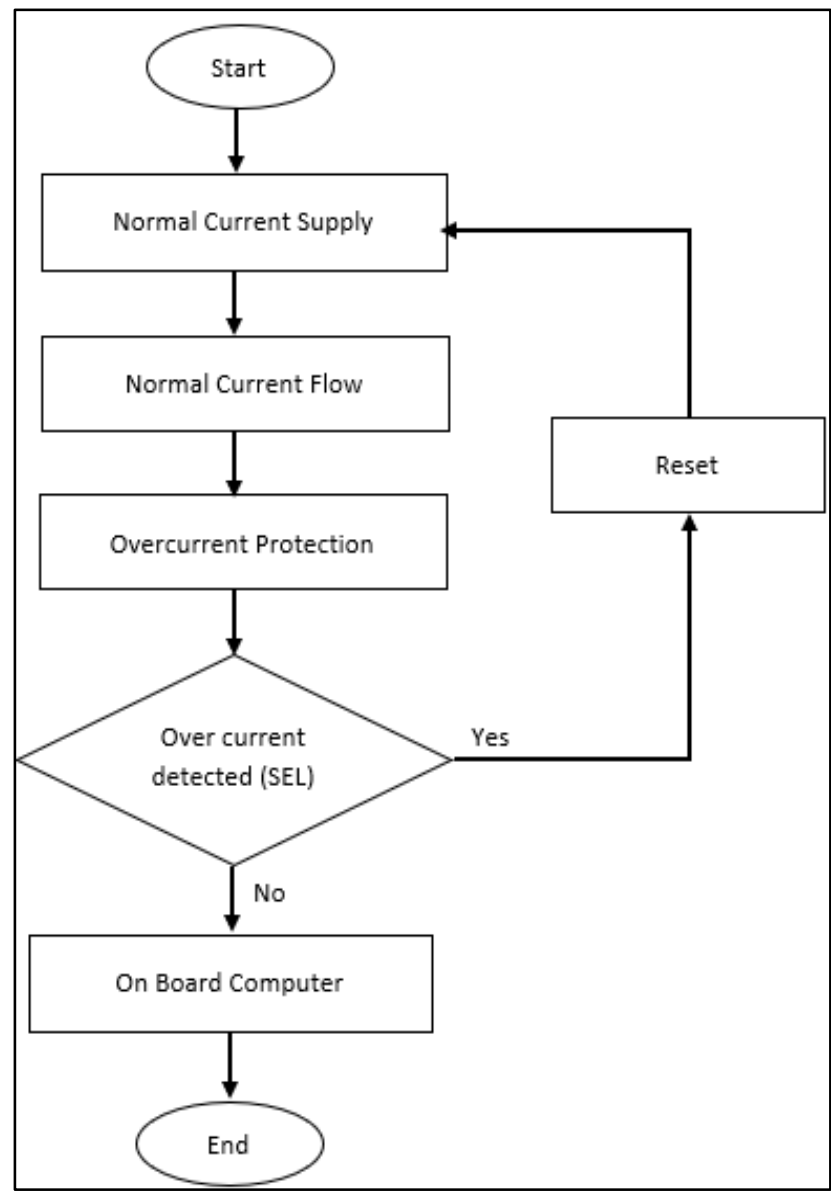

Figure 2. The flowchart of mitigating the SEL

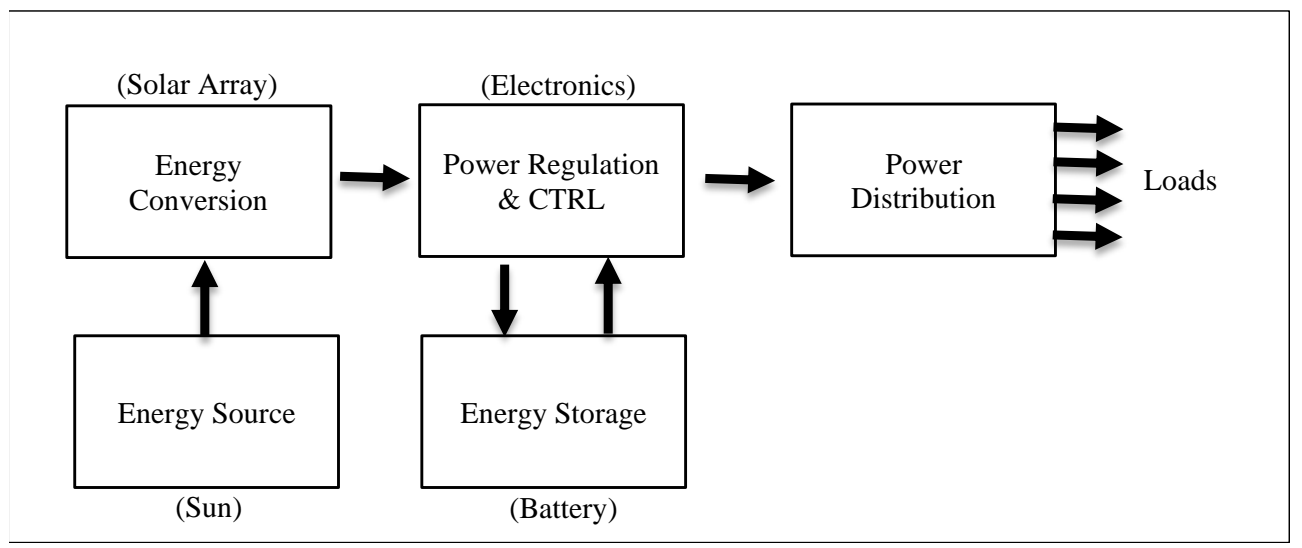

Figure 3. EPS standard block diagram

\subsection{Circuit study related to SEL}

The latch-up structure can be represented as a circuit behavioral model as shown in Figure 4 [27]. The model includes the junction resistance, the substrate resistance, the well resistance, and two crosscoupled BJTs. This model relies on measured resistance and BJT characterization values to accurately represent latch-up behavior. However, even without the specific resistances, the behavioral model is useful in exploring the effect of the resistor values on latch-up behavior, which is related to the spacing parameters under study in this work. 


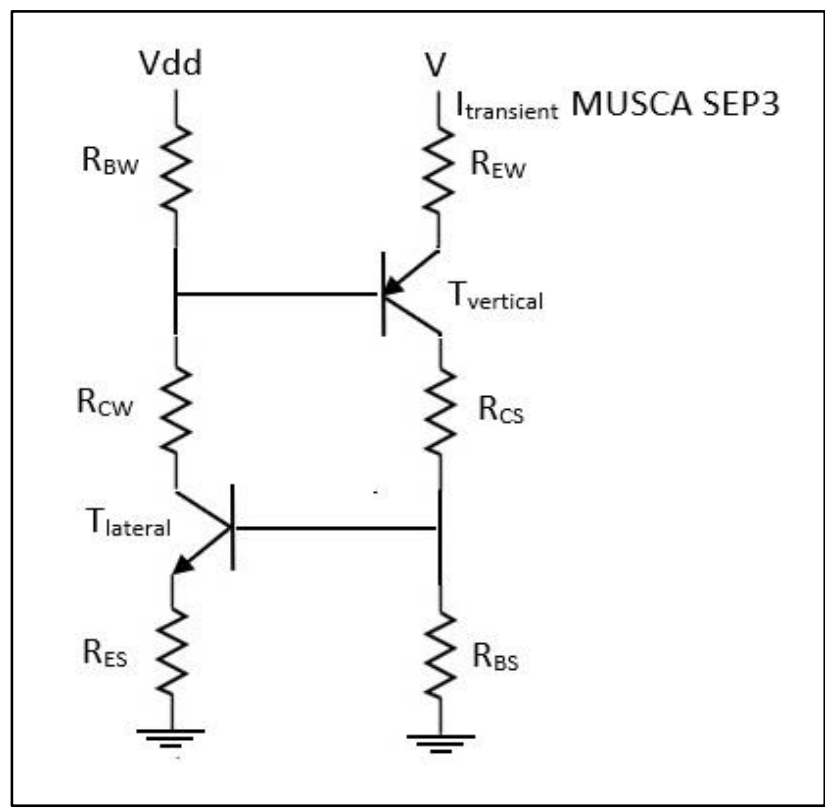

Figure 4. Latchup behavioral circuit model [27]

From model in Figure 4, the critical SEL parameter values for VHold and VTrig are calculated from (1) and (2):

$$
\begin{aligned}
& V_{\text {Trig }}=V_{D D}-V_{P N P t h}\left(1-\frac{R_{E W}}{R_{B S}}\right) \\
& V_{\text {Hold }}=V_{\text {Trig }}-\frac{R_{E W}\left(1-\frac{R_{E W} R_{E S}}{R_{B S} R_{B W}}\right)}{R_{E S}+R_{C W}+R_{B W}} V_{D D}
\end{aligned}
$$

$\mathrm{V}_{\text {Trig }}$ requires knowledge of the vertical parasitic bipolar junction transistor (BJT) threshold voltage, VPNPth, well emitter resistance, REW, and the substrate trigger resistance, RBS. VTrig represents the minimum required voltage to forward-bias the vertical parasitic BJT. VHold represents the level above which sustains the latch-up phenomenon. It depends on VTrig and a combination of the resistors in the model due to the feedback loop that sustains latch-up behavior [19].

\section{RESULTS AND DISCUSSION}

\subsection{Testing inject current}

Figure 5 shows the current increase observed during the proton test when SELs were observed. The three SELs included in this figure were observed at 5200, 5700 and $6000 \mathrm{sec}$, respectively. Before a SEL, the current consumption was near $40 \mathrm{~mA}$. It jumped to nearly $180 \mathrm{~mA}$ at the time of a SEL occurrence (see 5200 $\mathrm{sec}$ ). The only way to exit from the SEL condition was to turn the power on and off. The SELs were initialized by turning off the power to the test article at 5500, 5900 and $6100 \mathrm{sec}$, respectively. As the SELs occurred so quickly, it was difficult to see the nominal operation with a 40-mA current before the third SEL due to the limited data sampling rate.

\subsection{Spenvis simulation}

The testing using Spenvis is to see the activity of SEL in space. This reason why this test is to get the real value of SEL occurrence in real space area. The test has been set with Malaysia's satellite UiTMSAT-1. All kind of radiation effects that can affect the satellites orbiting in LEO are investigated and represented in graphical orm with the help of SPENVIS. We know that the radiation exposure of spacecraft in LEO is dependent on orbit inclinations and altitudes, the Figure 6 shows the of ionizing dose for UiTMSAT-1 in its own altitude inclination. The graph shows the ionizing dose effects on the aluminum shielding of the satellite, it also shows that the effect of electrons fades as the thickness of the shielding is increased but on the other hand the effect of proton has not much change. 


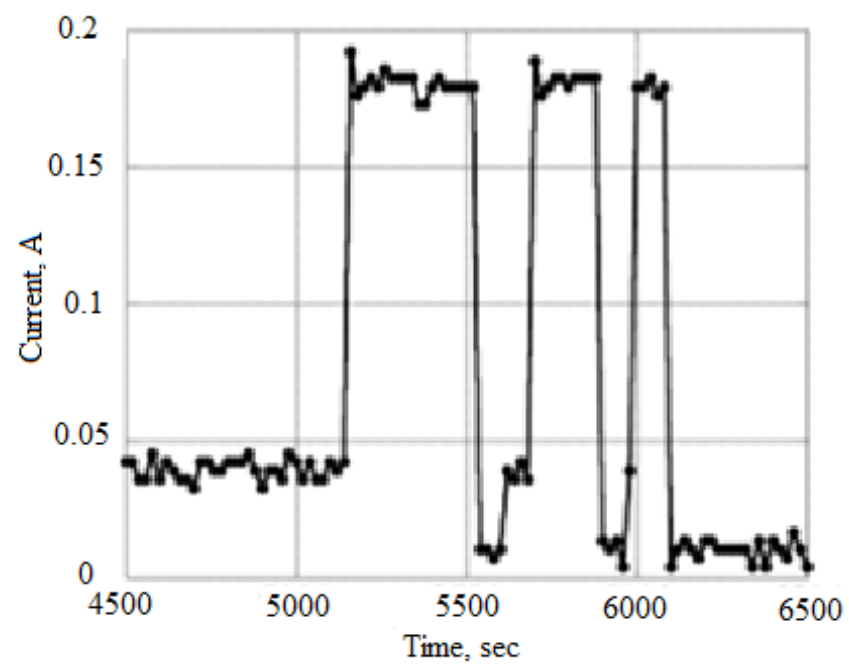

Figure 5. An example of consumption current jump when SEL was observed

Dose at Transmission Surface of AI Slab Shields

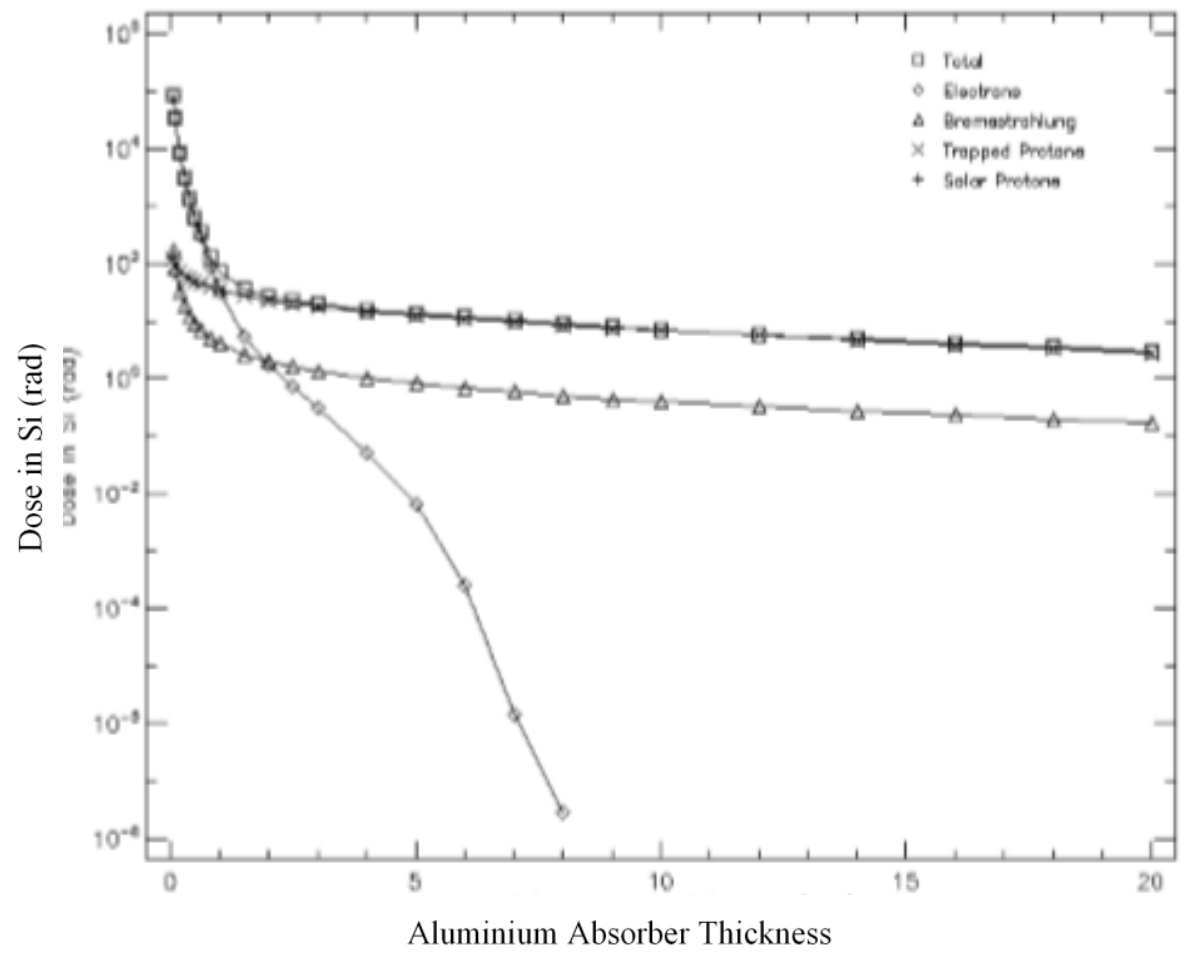

Figure 6. UiTMSAT-1 tested with ionizing dose effect

From the Figure 7 it is clear that the electrons and protons effect start at 10 rad dose and the electron effect was stopped at $8 \mathrm{~mm}$ of aluminium shielding. The data that tested using ionizing dose effect received from Spenvis only present of the single event upset (SEU). The Figure 7 also shows the SEU effects on UiTMSAT-1. In this Figure the effect of integral flux starts at almost $0.009 \mathrm{M} \mathrm{m}-2 \mathrm{sr}-1 \mathrm{~s}-1$ and the effect of differential flux starts at $0.002 \mathrm{M} \mathrm{m}-2 \mathrm{sr}-1 \mathrm{~s}-1$. For this also the integral and differential flux is stopped at $5 \times 102 \mathrm{MeV}$ of LET. 


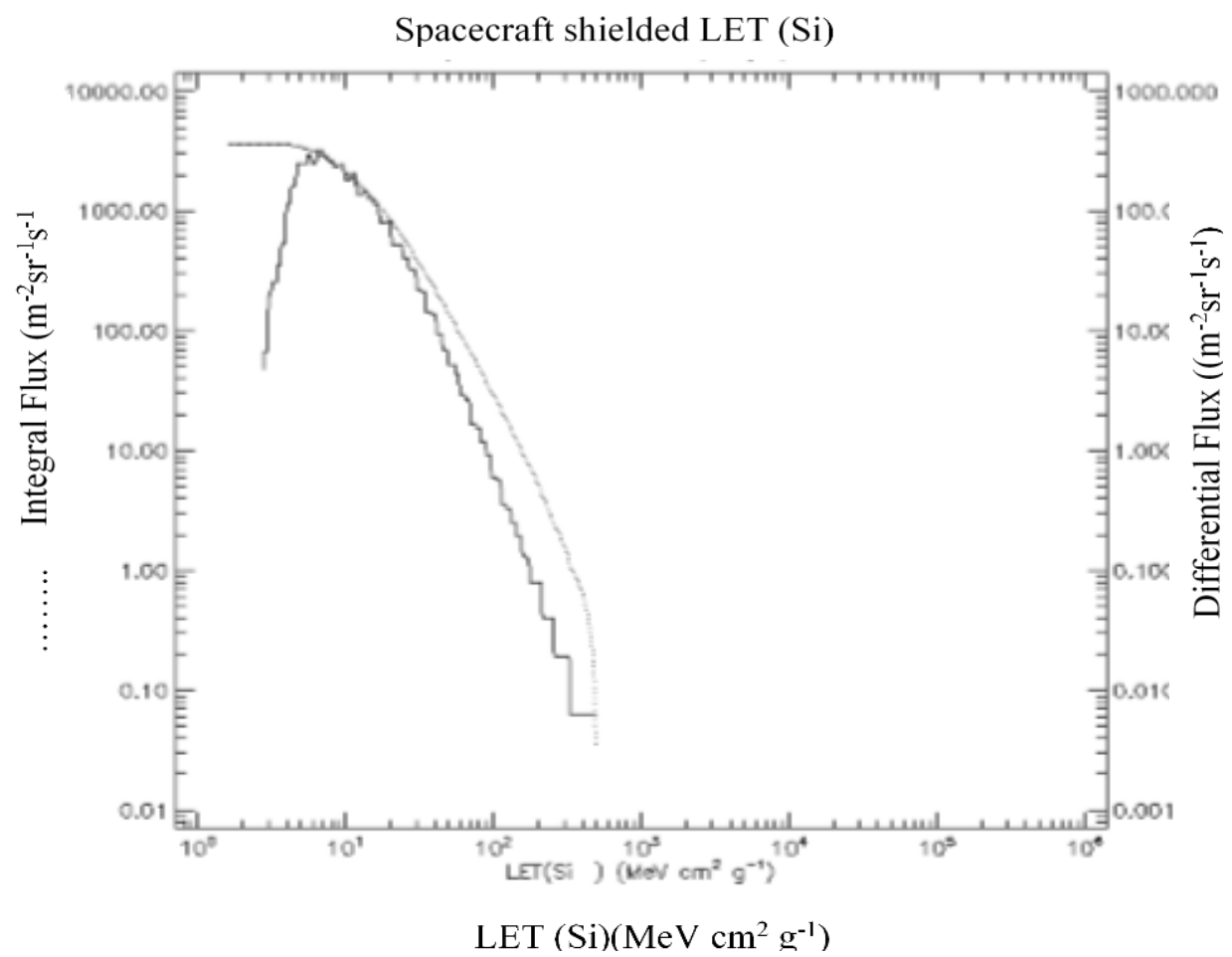

Figure 7. The event effect to UiTMSAT-1

\subsection{Mitigate the event}

The use of shield to reduce radiation and using software's to analyze the shield thickness and analysis of radiation tolerant circuits is essential. By increasing the thickness of aluminium shielding on satellites, we can reduce the radiation effects. Especially by increasing the shielding size we can reduce TID effects to a minimum because, the electrons causing TID have low penetration capacity. But other radiational effects cannot be stopped by just shielding, to avoid them the electronic components used in the satellites must undergo Radiation Hardening before going to space. Assigning safe orbital path for satellites can also reduce the radiation effects and this is because in the atmosphere at some regions' radiations are extreme.

\section{CONCLUSION}

This paper presents the SEL detection for nano-satellite external solar radiation mitigation system. The results showed from circuit tested and simulation are different output. Where in the current injection circuit testing was indicate the amount of SEL while in SPENVIS simulation, only SEU effecting was appeared. From this analysis shows both events can harm the OBC and cause the damage to the whole satellite. The mitigation of the SEE must be continuing the researches so that the mission carried can be done and received the desired outcome.

\section{ACKNOWLEDGEMENTS}

The authors would like to thank Faculty of Electrical Engineering, Universiti Teknologi MARA (UiTM) for their valuable support. This research is partly funded by the Malaysian Government through UiTM under 600-IRMI/5/3/GIP (0042/2019).

\section{REFERENCES}

[1] S. N. M. Rahim, J. Johari, S. A. Ence Ab Rahim and M. H. Jusoh, "Estimation of Communication Link on Ground Sensor Terminal (GST) System for Nanosatellite (UiTMSAT-1) Store-and-Forward Mission," 2018 IEEE 8th International Conference on System Engineering and Technology (ICSET), Bandung, pp. 108-111, 2018.

[2] S. N. Mohamad Rahim, et al, "Installation of UiTM's ground station-Connecting earth and space with amateur band communication," IOP Conference Series: Journal of Physics, vol. 1152, 2019. 
[3] S. N. Mohamad Rahim, et al, "Development of UiTMSAT-1: An Approach to Lean Satellite Concept," ASM Science Journal, vol. 12, no. 2, pp. 1-192, 2019.

[4] W. Suparta, "Space Weather Effects on Microelectronics Devices around the LEO Spacecraft Environments," Journal of Physics: Conference Series, vol. 539, no. 1, 2014.

[5] Samir A. Rawashdeh, "Attitude Analysis of Small Satellites Using Model-Based Simulation," International Journal of Aerospace Engineering, vol. 2019, no. 3020581, pp. 1-11, 2019.

[6] Md. Mahbubur Rahman, et al, "Analysis of Radiation Environment and its Effect on Spacecraft in Different Orbits," Conference: international Astronautical Congress (IAC2017), Adelaide, Australia, vol. 1, pp. 8073-8079, 2017.

[7] Juan Sebastián Triana, et al, "Identification of Design Considerations for Small Satellite Remote Sensing Systems in Low Earth Orbit," Journal of Aerospace Technology and Management, vol. 7, no. 1, pp. 121-134, 2015.

[8] Cristóbal Nieto-Peroy and M. Reza Emami, "CubeSat Mission: From Design to Operation," Applied Science, vol. 9, no. 15, 2019.

[9] Doug Sinclair, Jonathan Dyer, "Radiation Effectsand COTS Parts in SmallSats," 27th Annual AIAA/USU Conference on small satellites, pp. 1-12, 2013.

[10] H. N. Becker, T. F. Miyahira and A. H. Johnston, "Latent damage in CMOS devices from single-event latchup," in IEEE Transactions on Nuclear Science, vol. 49, no. 6, pp. 3009-3015, Dec 2002.

[11] D. Del Corso, C. Passerone, L. Reyneri, C. Sansoe, S. Speretta and M. Tranchero, "Design of a University Nano-Satellite: the PiCPoT Case," in IEEE Transactions on Aerospace and Electronic Systems, vol. 47, no. 3, pp. 1985-2007, July 2011.

[12] Takahiro Tomioka, et al, "Screening of nanosatellite microprocessors using californium single-event latch-up test results," Acta Astronautica, vol. 126, pp. 334-341, 2016.

[13] Hyrso Villela, et al, "Towards the Thousandth CubeSat: A Statistical Overview," International Journal of Aerospace Engineering, vol. 2019, article id. 5063145, pp. 1-13, 2019.

[14] Chang Cai, et al, "SEE Sensitivity Evaluation for Commercial n16 nm SRAM-FPGA," Electronics, vol. 8, no. 12, pp. 1-12, 2019.

[15] P. J. Botma, A. Barnard and W. H. Steyn, "Low cost fault tolerant techniques for nano/pico-satellite applications," 2013 Africon, Pointe-Aux-Piments, pp. 1-5, 2013.

[16] Jan Budroweit, et al, "Heavy Ion Induced Single Event Effects Characterization on an RF-Agile Transceiver for Flexible Multi-Band Radio Systems in NewSpace Avionics," Aerospace, vol. 7, no. 2, 2020.

[17] Pengwei Li, Xiaoyun Fu, Lei Luo, Qingkui Yu, "A New Analyzing Method of Single Event Latch-Up Protection Circuit Based on Current Comparing and Its Performance Verification," Journal of Modern Physics, vol. 5, no. 6, pp. 387-393, 2014.

[18] A. N. Nikicio, W. Loke, H. Kamdar and C. Goh, "Radiation analysis and mitigation framework for LEO small satellites," 2017 IEEE International Conference on Communication, Networks and Satellite (Comnetsat), Semarang, pp. 59-66, 2017.

[19] Todd, Benjamin and Uznanski, Slawosz, "Radiation Risks and Mitigation in Electronic Systems," arXiv, pp. 245-263, 2016.

[20] Jan Budroweit Mattis Paul Jaksch Maciej Sznajder, "Proton Induced Single Event Effect Characterization on a Highly Integrated RF-Transceiver,” Electronics, vol. 8, no. 5, pp. 1-20, 2019.

[21] Maham Kamil Naji, Alaa Desher Farhood, Adnan Hussein Ali, "Novel design and analysis of RF MEMS shunt capacitive switch for radar and satellite communications," Indonesian Journal of Electrical Engineering and Computer Science (IJEECS), vol. 15, no. 2, pp. 971-978, Aug 2019.

[22] Akihiro Nagata, et al, "Development of a Support System for Radiation Resistance Testing," Transactions Of The Japan Society For Aeronautical and Space Sciences, Aerospace Technology Japan, vol. 17, no. 2, pp. 263-269, 2019.

[23] Chef, Samuel et al. "VLSI for Space Applications: Single Event Effect Investigation and Optical Analysis on an Integrated Laser Platform," Conference: 43rd International Symposium for Testing and Failure Analysis (ISTFA), At Pasadena, CA, USA, 2017.

[24] Andrew Sternberg, et al, "Using Pulsed lasers as a Diagnostic tool for radiation-induces single Event latchup," 31st Annual AIAA/USU conference on small satellites, 2017.

[25] A. Al Youssef, L. Artola, S. Ducret, G. Hubert and F. Perrier, "Investigation of Electrical Latchup and SEL Mechanisms at Low Temperature for Applications Down to $50 \mathrm{~K}$," in IEEE Transactions on Nuclear Science, vol. 64, no. 8, pp. 2089-2097, Aug 2017.

[26] Linh Ta Phuong, et al, "A microwave active filter for nanosatellite'sreceiver front-ends at S-band," International Journal of Electrical and Computer Engineering (IJECE), vol. 9, no. 2, pp. 973-981, April 2019.

[27] L. Artola, G. Hubert and T. Rousselin, "Single-Event Latchup Modeling Based on Coupled Physical and Electrical Transient Simulations in CMOS Technology," in IEEE Transactions on Nuclear Science, vol. 61, no. 6, pp. 3543-3549, Dec 2014. 